\title{
Spatially resolved exciton trapping in a voltage-controlled lateral superlattice
}

Cite as: Appl. Phys. Lett. 73, 154 (1998); https://doi.org/10.1063/1.121740

Submitted: 21 January 1998 - Accepted: 09 May 1998 Published Online: 07 July 1998

S. Zimmermann, G. Schedelbeck, A. O. Govorov, et al.

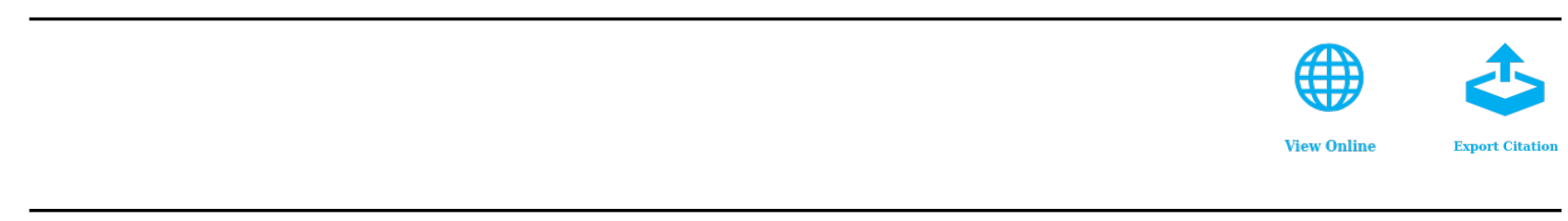

\section{ARTICLES YOU MAY BE INTERESTED IN}

\section{Excitons in electrostatic traps}

Journal of Applied Physics 99, 066104 (2006); https://doi.org/10.1063/1.2181276

Electric-field-induced exciton transport in coupled quantum well structures Applied Physics Letters 67, 232 (1995); https://doi.org/10.1063/1.114677

Drift mobility of long-living excitons in coupled GaAs quantum wells

Applied Physics Letters 89, 052108 (2006); https://doi.org/10.1063/1.2267263

母QBLOX

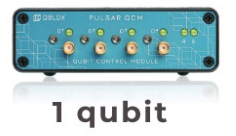

Shorten Setup Time Auto-Calibration More Qubits

Fully-integrated Quantum Control Stacks Ultrastable DC to $18.5 \mathrm{GHz}$ Synchronized $<<1$ ns Ultralow noise

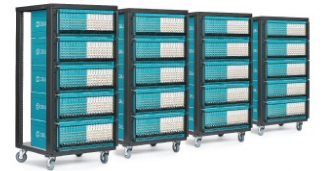

100s qubits

visit our website > 


\title{
Spatially resolved exciton trapping in a voltage-controlled lateral superlattice
}

\author{
S. Zimmermann, ${ }^{\text {a) }}$ G. Schedelbeck, ${ }^{\text {b) }}$ A. O. Govorov, ${ }^{\text {c) }}$ A. Wixforth, J. P. Kotthaus, \\ M. Bichler, ${ }^{\text {b) }}$ W. Wegscheider, ${ }^{\text {b) }}$ and G. Abstreiter ${ }^{\text {b) }}$ \\ Sektion Physik der LMU, Geschw.-Scholl-Platz 1, D-80539 München, Germany
}

(Received 21 January 1998; accepted for publication 9 May 1998)

\begin{abstract}
With spatially resolved photoluminescence experiments we demonstrate voltage-controlled trapping of excitons in a submicron scale lateral potential superlattice imposed on a semiconductor quantum well. The potential modulation is achieved by two interdigitated field-effect electrodes on top of the sample surface. Both parallel and vertical electric field components strongly modify the optical properties of the quantum well. We show that the lateral modulation of the strength of the quantum confined Stark effect results in an effective lateral exciton potential that can be probed by spatially resolved measurements of the excitonic luminescence. We demonstrate that excitons may be confined to the regions of strongest vertical fields, in which the effective exciton energy is lowest. Spatial resolution of the observed photoluminescence signal allows for a qualitative understanding of the exciton transport and trapping process. (C) 1998 American Institute of Physics.
\end{abstract}

[S0003-6951(98)01428-4]

Electro-optic interactions involving excitons in semiconductor microstructures and devices are attracting much interest over the last years. ${ }^{1}$ The effect of electric fields on the optical properties of semiconductor heterostructures is an especially intense subject of investigations. This is motivated by the fact that electric fields can substantially modify the optical properties and, hence, may be used for the design of possible new optoelectronic devices. It is well known that an electric field perpendicular to the plane of the quantum well (QW) results in the occurrence of the quantum confined Stark effect (QCSE) ${ }^{2}$ causing a redshift of the exciton energy as compared to the unperturbed case. Field components parallel to the plane of the QW induce the two-dimensional Franz-Keldysh effect. ${ }^{3,4}$ Consequently, it is interesting to study quantum well excitons in laterally varying electrostatic potentials where the optical properties of a quantum well can be modulated on a submicron scale. Moreover, a possible tunability of the potential landscape allows for a deliberate control of the optical properties both in space and time. Earlier attempts to confine QW excitons to well defined submicron regions employed static methods like strain, ${ }^{5}$ interdiffusion of a barrier material, ${ }^{6}$ and the preparation of selforganized lateral structures. ${ }^{7}$ As has been shown before, dynamic transport of QW excitons can be achieved via strong spatially varying electric fields due to the QCSE. ${ }^{8}$

Here we directly demonstrate tunable transport and trapping ${ }^{9,10}$ of neutral excitons into submicron wide channels employing a spatially resolved photoluminescence (PL) experiment. The mechanism responsible for tunable exciton localization is based on the following: Electric fields $F_{z}$ normal to the plane of the quantum well polarize the exciton and therefore lead to an excitonic energy shift, the QCSE. In comparatively wide quantum wells this shift can be much larger than the linewidth of the corresponding excitonic PL.

\footnotetext{
${ }^{a)}$ Electronic mail: Stefan.Zimmermann @ physik.uni-muenchen.de

${ }^{b)}$ Also at: Walter-Schottky-Institut der TU München, Coulombwall 1, D85748 Garching, Germany.

c)Also at: Institut of Semiconductor Physics, 630090 Novosibisk, Russia.
}

In the linear regime of the QCSE it can be approximated by $\Delta E \approx-d F_{z}$, where $d$ denotes the field-induced dipole moment of the exciton. ${ }^{11}$ If the strength of the vertical electric field is dependent on the lateral coordinate $x$ in the direction of the QW plane, a force $\mathscr{F}_{x}=-d \Delta E(x) / d x$ is acting on the excitons in this direction and the laterally modulated energy shift $\Delta E(x)$ converts into an effective exciton potential modulation $U_{\text {eff }}(x)$. Thus, excitons will drift into the minima of the effective potential and a tunable exciton trap can be realized.

For this purpose, we employ an extremely versatile semiconductor system ${ }^{4,12}$ as sketched in Fig. 1. Interdigitated top gate electrodes and a doped back contact at some distance below the QW are used to apply different negative bias voltages $V_{1}$ and $V_{2}$ between the gates and the back contact. ${ }^{13}$ This imposes different normal electric fields under the stripes of gate 1 and gate 2 and hence creates a widely tunable lateral potential superlattice in the plane of the QW as depicted in Fig. 1. The induced spatial modulation of the QCSE generates the effective exciton potential $U_{\text {eff }}$ which confines photogenerated excitons to regions under the more negatively biased gate stripes. Here, the vertical electric field

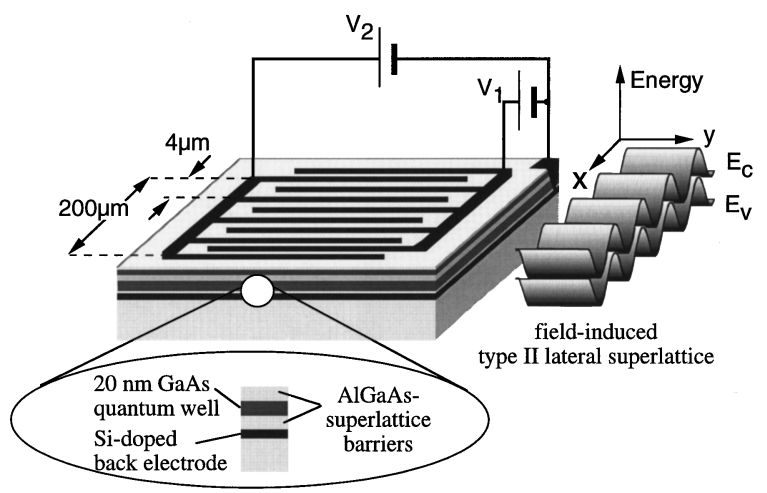

FIG. 1. Interdigitated gate structure on the surface of a GaAs heterostructure with a back electrode underneath the quantum well $(\mathrm{QW})$. Two different voltages $V_{1}$ and $V_{2}$ applied with respect to the back electrode induce a lateral superlattice in the QW plane and, hence, a pattern of strongly varying vertical and lateral electric fields. 
components are strongest and the exciton potential energy is minimal. As the exciton diffusion length ${ }^{14}$ as well as the diffraction limited spatial resolution of our PL measurements are of the order of $1 \mu \mathrm{m}$, we use an interdigitated gate structure with period $a=2 \mu \mathrm{m}$, stripe width $w=1.1 \mu \mathrm{m}$ and a gap of $d=0.9 \mu \mathrm{m}$ between the stripes. Hence we are able to investigate with spatially resolved measurements the redistribution of excitons caused by diffusion and field-induced drift and to confirm in this way directly our model of the effective exciton potential. ${ }^{9,10}$

The AlAs-GaAs heterostructure used in our experiments is grown by molecular beam epitaxy and consists of a $\mathrm{Si}$ doped back electrode $\left(N_{D}=4 \times 10^{18} \mathrm{~cm}^{-3}\right)$ grown on an undoped GaAs buffer and a 20-nm-wide undoped QW sandwiched between two short-period AlAs-GaAs superlattice barriers. The QW and the back electrode are separated from the surface by $d_{\mathrm{QW}}=60 \mathrm{~nm}$ and $d_{\mathrm{BE}}=390 \mathrm{~nm}$, respectively. The 10-nm-thick interdigitated gate electrodes $(A$ $=200 \times 200 \mu \mathrm{m}^{2}$ ) are defined by means of electron beam lithography on top of the sample consists of semi-transparent titanium $(d=10 \mathrm{~nm})$. Our experiments are performed in a low temperature optical cryostat at $T=6 \mathrm{~K}$ to assure PL of excitonic origin. The QW excitons are generated using a dye laser beam $(E=1.58 \mathrm{eV}$ and $P=5 \mu \mathrm{W})$ being focused on the sample to a spot of $1 \mu \mathrm{m}$ diameter by means of a microscope objective. The cryostat can be moved with respect to the optical axis of the setup via a micrometer translation stage (accuracy of reposition $\approx 50 \mathrm{~nm}$ ) to scan the laser spot across the sample. The outgoing light of the PL is analyzed in a triple grating spectrometer using a charged coupled device (CCD) camera as a detector. To also ensure a spatially resolved detection area on the sample, we place a pinhole in the focal plane of a Galilei telescope placed into the optical path. This pinhole can be moved independently from the sample and the optical axis and allows for a spatial offset of the detection area relative to the excitation area.

In Fig. 2 we show a set of such spatially resolved PL spectra. Here, excitation and detection area are kept identical and are scanned simultaneously across the sample in the direction normal to the gate stripes ( $x$ direction). Every $\Delta x=100 \mathrm{~nm}$ a PL spectrum is taken. In Fig. 2(a) equal gate voltages $V_{1}=V_{2}=-0.5 \mathrm{~V}$ are applied to the interdigital gate structure, whereas in Fig. 2(b) the gate voltages are chosen to be different $\left(V_{1}=-0.7 \mathrm{~V}\right.$ and $\left.V_{2}=-0.3 \mathrm{~V}\right)$. To visualize the topography of the sample, we coded the spectra with a grey scale according to their site of origin corresponding to the location of the gate stripes. For the case of equal gate bias to both gate electrodes (a), we detect a nearly homogeneous distribution of the exciton PL energy and intensity at $E=1.515 \mathrm{eV}$. The gates thus essentially provide a laterally homogeneous normal electric field and strength of the QCSE across the QW. This is reflected in the PL energy which is red shifted as compared to the one under flat band conditions $(E=1.526 \mathrm{eV}) .{ }^{10}$ The small spatial intensity modulations can be explained by the reduced optical transmission in the regions of the semitransparent gate stripes.

However, if two different negative voltages $V_{1}=-0.7$ $\mathrm{V}$ and $V_{2}=-0.3 \mathrm{~V}$ are applied to the gates, we observe a significant spatial dependence of the PL energy and intensity [Fig. 2(b)]. The spatial energy dispersion is caused by the
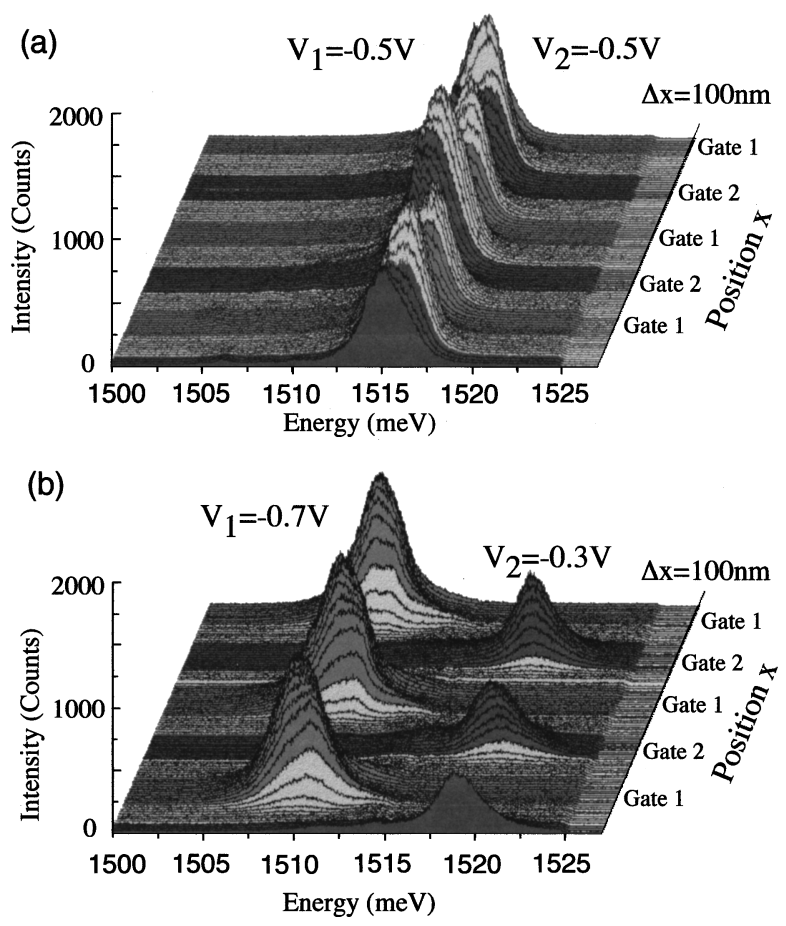

FIG. 2. Spatially resolved PL measurement for different gate voltage differences $\Delta V=V_{1}-V_{2}$. (a) $V_{1}=V_{2}=-0.5 \mathrm{~V}$ and (b) $V_{1}=-0.7 \mathrm{~V}, V_{2}$ $=-0.3 \mathrm{~V}$. The overlapping PL excitation and detection areas of $1 \mu \mathrm{m}$ diam are scanned simultaneously across the sample in steps of about $100 \mathrm{~nm}$ in the direction $x$ perpendicular to the gate stripes. The approximate position of the gate stripes is indicated in gray shades.

different vertical electric field components below the stripes of gate 1 and gate 2 . This spatially varying electric field causes a different strength of the local QCSE and alternates spatially with the gate period. The PL originating from regions below the stripes of gate 1 of energy $1.508 \mathrm{eV}$ is red shifted, whereas the PL from regions below the stripes of gate 2 of energy $1.518 \mathrm{eV}$ is blue shifted as compared to (a). The small energetic shifts of all peaks towards the edges of the gate stripes in Figs. 2 and 3 are caused by a combination of a slight lateral modulation of both the light transmission and the vertical field strength. ${ }^{9}$ The intensity of the red shifted PL (gate 1, gray) is larger than the blue shifted PL (gate 2, dark gray) which indicates an accumulation of excitons in regions of strongest vertical electric fields below the stripes of gate 1. It should be noted that in the regions between the gate stripes (light gray) the PL intensity completely vanishes. Excitons created here are either ionized by strong lateral electric fields between the gate stripes $^{9,10}$ or removed from this region by a drift into the regions of strongest vertical electric fields before they recombine. This drift induced by the gradients of the lateral effective exciton potential will be addressed explicitly in the following.

In Fig. 3, we investigate the exciton drift from regions of different effective exciton potential. This is realized by keeping the PL excitation area fixed and scanning the detection area relative to it. Approximately every $450 \mathrm{~nm}$ a PL spectrum is taken, covering an area corresponding to approximately two neighboring stripes of gates 1 and 2. Only a moderate gate voltage difference $\left(V_{1}=-0.6 \mathrm{~V}\right.$ and $V_{2}$ $=-0.4 \mathrm{~V}$ ) between the gate stripes is used in order to prevent exciton ionization in the high lateral field regions between the gate stripes. To generate high exciton densities in 
(a)

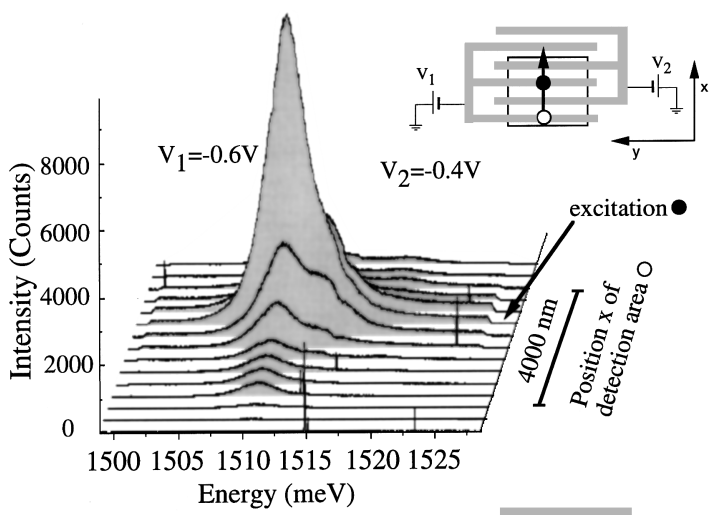

(b)

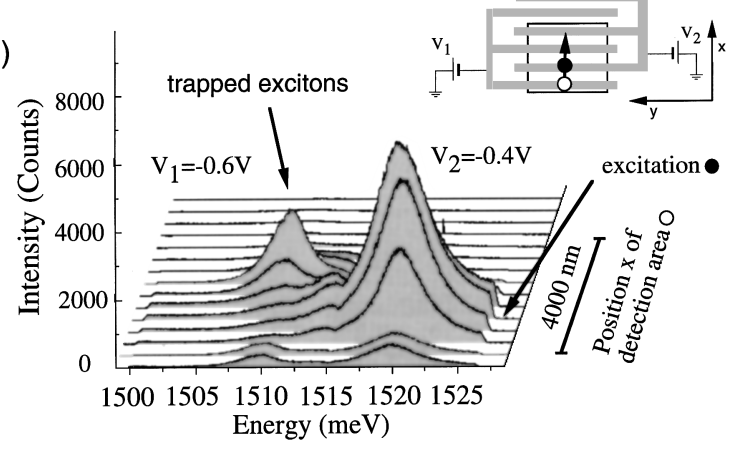

FIG. 3. Spatially resolved measurement of the exciton drift. For (a) and (b), the PL excitation area is kept fixed as indicated in the insets. The detection area is scanned in lateral $x$ direction normal to the gate stripes over a length of approximately $4 \mu \mathrm{m}$. The gate voltages applied are $V_{1}=-0.6 \mathrm{~V}$ and $V_{2}=-0.4 \mathrm{~V}$. (a) Excitation on a gate stripe with potential $V_{1}$. (b) Excitation on a gate stripe with potential $V_{2}$.

the QW, we operate at an elevated excitation power of the laser $(P=65 \mu \mathrm{W})$ which is focused to $1 \mu \mathrm{m}$ diam onto the sample surface.

In Fig. 3(a), the excitation area is located on a stripe of gate 1 with a more negative gate bias $V_{1}=-0.6 \mathrm{~V}$ as compared to gate $2\left(V_{2}=-0.4 \mathrm{~V}\right)$. The detection area is then scanned across the sample along the $x$-direction normal to the gate stripes. The spectra exhibit an intense PL line at $E$ $=1.510 \mathrm{eV}$, if the excitation and detection area completely overlap. If the detection area is scanned to regions corresponding to the neighboring gate stripes and beyond, the PL intensity vanishes, the residual signal being mainly caused by stray light. From this finding, we conclude that the recombining excitons are located in the potential minima below the stripes of gate 1 .

The situation changes when we excite the PL on a stripe of gate 2 [Fig. 3(b)]. If the positions of the detection area and the excitation area are identical, the PL spectra exhibit an intense line at $E=1.51 \mathrm{eV}$. The higher energetic position of the line as compared to (a) is understood in terms of the less pronounced QCSE under gate 2. Furthermore, its intensity is smaller than in (a) as the effective exciton potential is in the region investigated of repulsive form. This allows the excitons to easily leave the excitation area by diffusion and drift. By scanning the detection area into regions between the gate stripes, we observe a strong decrease of the PL intensity. However, if the detection area reaches the regions of the neighboring stripes of gate 1 with the more negative potential, the PL intensity rises again at an energy of $E$ $=1.510 \mathrm{eV}$. We interpret this line as a PL of excitons that are located under gate 1 [cf. Fig. 3(b)]. We hence can con- clude that the effective exciton potential induced by the gate voltage difference leads, in fact, to a redistribution of excitons, such that they are trapped and localized in regions of strongest vertical electrical fields under the more negative gate. As we do not observe any PL emitted from regions between the gate stripes, we conclude that the excitons drift into the potential minima in a time shorter than their radiative life time of about $1 \mathrm{~ns} .{ }^{15}$ Furthermore, scanning the detection area to more remote locations from the excitation area in direction of positive $x$ does not reveal any PL. This is consistent with the fact that the excitons do not drift further than one-half period of the interdigitated gate. They are efficiently trapped in regions under the next neighbor gate stripe.

In summary, we have probed the spatial distribution and redistribution of photogenerated excitons in a semiconductor heterostructure with a periodic potential modulation in the plane of the quantum well. The spatially varying electric fields generated by an interdigitated gate electrode lead to a spatial variation of the strength of the quantum confined Stark effect. This results in an effective exciton potential such that the excitons become confined in regions of local potential minima. Employing a spatially resolved PL technique we directly observe the drift of excitons into these potential minima and their accumulation in the voltage tunable trap.

This work was supported by the Sonderforschungsbereich 348 of the Deutsche Forschungsgemeinschaft. One of the authors (A.O.G.) gratefully acknowledges support by the Volkswagen-Stiftung and the FOROPTO project of the Bayerische Forschungsstiftung.

${ }^{1}$ Optics of Semiconductor Nanostructures, edited by F. Henneberger, S. Schmitt-Rink, and E. O. Göbel (Akademie, Berlin, 1993).

${ }^{2}$ D. A. B. Miller, D. S. Chemla, T. C. Damen, A. C. Gossard, W. Wiegmann, T. H. Wood, and C. A. Burrus, Phys. Rev. B 32, 1043 (1985).

${ }^{3}$ W. Franz, Z. Naturforsch. A 13, 484 (1958); L. V. Keldysh, Sov. Phys. JETP 7, 7878 (1958)

${ }^{4}$ A. Schmeller, W. Hansen, J. P. Kotthaus, G. Tränkle, and G. Weimann, Appl. Phys. Lett. 64, 330 (1994).

${ }^{5}$ K. Kash, J. M. Worlock, M. D. Sturge, P. Grabbe, J. P. Harbison, A. Scherer, and P. S. D. Lin, Appl. Phys. Lett. 53, 782 (1988); Y. Gu, M. D. Sturge, K. Kash, N. Watkins, B. P. Van der Gaag, A. S. Gozdz, L. T. Florez, and J. P. Harbison, ibid. 70, 1733 (1997).

${ }^{6}$ K. Brunner, G. Abstreiter, M. Walther, G. Böhm, and G. Tränkle, Surf. Sci. 267, 218 (1992).

${ }^{7}$ J. Y. Marzin, J. M. Gerard, A. Izrael, D. Barrier, and G. Bastard, Phys. Rev. Lett. 73, 716 (1994)

${ }^{8}$ M. Hagn, A. Zrenner, G. Böhm, and G. Weimann, Appl. Phys. Lett. 67, 232 (1995).

${ }^{9}$ A. Schmeller, A. Govorov, W. Hansen, J. P. Kotthaus, W. Klein, G. Böhm, G. Tränkle, and G. Weimann, Proceedings of the 22nd International Conference on the Physics of Semiconductors, Vancouver, Canada, 1994 (World Scientific, Singapore, 1995), Vol. 2, p. 1727.

${ }^{10}$ S. Zimmermann, A. O. Govorov, W. Hansen, J. P. Kotthaus, M. Bichler and W. Wegscheider, Phys. Rev. B 56, 13414 (1997).

${ }^{11}$ L. Vina, E. E. Mendez, W. I. Wang, L. L. Chang, and L. Esaki, J. Phys. C 20, 2803 (1987)

${ }^{12}$ H. Drexler, W. Hansen, S. Manus, J. P. Kotthaus, M. Holland, and S. P. Beaumont, Phys. Rev. B 49, 14074 (1994); H. Drexler, W. Hansen, J. P. Kotthaus, M. Holland, and S. P. Beaumont, Appl. Phys. Lett. 64, 2270 (1994).

${ }^{13}$ We then operate in the nondegenerate regime, where the quasi Fermi levels remain in the effective band gap.

${ }^{14}$ W. Heller, A. Filoramo, Ph. Roussignol, and U. Bockelmann, Solid-State Electron. 40, 725 (1996).

${ }^{15}$ J. Feldmann, G. Peter, E. O. Göbel, P. Dawson, K. Moore, C. Foxon, and R. J. Elliot, Phys. Rev. Lett. 59, 2337 (1987). 